\title{
The nonlinear development of the relativistic two-stream instability
}

\author{
I. Hawke ${ }^{1}$, G. L. Comer ${ }^{2}$, N. Andersson ${ }^{1}$ \\ E-mail: I.Hawke@soton.ac.uk \\ ${ }^{1}$ Mathematical Sciences, University of Southampton, Southampton, SO17 1BJ, \\ $\mathrm{UK}$ \\ ${ }^{2}$ Department of Physics \& Center for Fluids at All Scales, Saint Louis \\ University, St. Louis, MO, 63156-0907, USA
}

\begin{abstract}
The two-stream instability has been mooted as an explanation for a range of astrophysical applications from GRBs and pulsar glitches to cosmology. Using the first nonlinear numerical simulations of relativistic multispecies hydrodynamics we show that the onset and initial growth of the instability is very well described by linear perturbation theory. In the later stages the linear and nonlinear description match only qualitatively, and the instability does not saturate even in the nonlinear case by purely ideal hydrodynamic effects.
\end{abstract}




\section{Introduction}

The two-stream instability occurs generically when two coupled, inter-penetrating fluids have a sufficiently large relative velocity. The expectation is that the instability arises when a perturbation appears to move in different directions with respect to each fluid. Originally studied in magnetized plasmas modeled by the collisionless Vlasov-Maxwell equations [1, 2], where a detailed understanding of the full mode spectrum exists (see e.g. [3]), it is only recently (see [4]) that it has been studied in the context of pure relativistic hydrodynamics. The well-understood plasma instability has astrophysical applications in GRBs (see e.g. [3]) and the pulsar emission mechanism $([5,6])$, whilst the hydrodynamic instability has been suggested as a key mechanism behind pulsar glitches $([7,8])$ and is of relevance in the cosmological context [9].

Most existing explorations of the two-stream instability have focused on its development in the linear regime, as - in astrophysically interesting applications such as pulsar glitches - the instability is expected to saturate at relatively low amplitude. However, the mechanism by which the instability saturates is not clear. The obvious possibility is that non-ideal effects such as shear viscosity will stop the growth. However, given the difficulties in constructing a consistent and stable relativistic non-ideal theory this avenue can only be pursued phenomenologically. An alternative possibility is that the instability saturates due to nonlinear effects within the framework of the ideal theory, possibly via the generation of internal shocks converting the unstable modes to heat. A final possibility that is unlikely to be generically applicable is that the fluids may be dynamically driven out of the instability window, perhaps by external forces or non-local effects, as happens in the cosmological case $([9])$.

In this paper we will study the nonlinear development of the relativistic hydrodynamic two-stream instability in simplified cases using numerical simulations. For reasons detailed later we are not able to consider shock propagation. However, the simulations can investigate if shock formation is a possibility, or whether the high frequency oscillations dominate, at which point we would expect non-ideal effects to be important. Our results suggest that when the instability is triggered by small perturbations the two-stream instability grows until the solution is dominated by high frequency oscillations. By comparing with linearized time-domain solutions we see that the nonlinear coupling has only a small effect, and is insufficient to saturate the instability.

\section{The system}

We consider the system of relativistic multiple fluids as introduced by Carter in [10] and detailed in the review of Andersson and Comer [11]. Here the notation largely follows [11], assumes the existence of a spacetime metric $g_{a b}$ of signature -+++ , uses Roman letters from the start of the alphabet $-a, b, c, \ldots-$ as 4 -spacetime indices, and from the middle $-i, j, k, \ldots-$ as 3 -space indices in the $3+1$ split. The characters $\mathrm{X}, \mathrm{Y}, \mathrm{Z}$ will be used as labels indicating the different fluids, or different species, which will not be implicitly summed over except where explicitly stated. Units where the speed of light $c=1$ are used throughout. 


\subsection{General form}

The basic fluid quantities are the number density currents $n_{\mathrm{X}}^{a}$ where $\mathrm{X}$ is a species label. Here we do not consider reactions or particle creation, meaning that the number densities are conserved. Hence the currents obey the continuity equations

$$
\nabla_{a} n_{\mathrm{X}}^{a}=0 .
$$

The system is closed using the master function $\Lambda$. The equations of motion - the Euler equations - follow by minimizing the action defined using this master function. The master function is defined in terms of all possible scalar invariants $n_{\mathrm{XY}}^{2}=-g_{a b} n_{\mathrm{X}}^{a} n_{\mathrm{Y}}^{b}$. The shorthand notation $n_{\mathrm{XX}}^{2} \equiv n_{\mathrm{X}}^{2}$ is often used. The Euler equations are written in terms of the conjugate momenta $\mu_{a}^{\mathrm{x}}$, defined by

$$
\mu_{a}^{\mathrm{X}}=\frac{\partial \Lambda}{\partial n_{\mathrm{X}}^{a}}=-2 \frac{\partial \Lambda}{\partial n_{\mathrm{X}}^{2}} n_{a}^{\mathrm{X}}-\sum_{\mathrm{Z} \neq \mathrm{X}} \frac{\partial \Lambda}{\partial n_{\mathrm{XZ}}^{2}} n_{a}^{\mathrm{Z}} .
$$

Using these definitions the Euler equations follow as

$$
f_{b}^{\mathrm{X}} \equiv 2 n_{\mathrm{X}}^{a} \nabla_{[a} \mu_{b]}^{\mathrm{X}}=0
$$

We note that the Euler equations are not written in balance law form. For a single fluid, or for the total number current in the case of multiple fluids, we can use the identity $\mu_{a} \nabla_{b} n^{a}=\nabla_{b} \Lambda$ to show that (3) is equivalent to the standard balance law form. In the general multifluid case a similar manipulation is only possible if for every species there is a potential $\Lambda_{\mathrm{X}}$ such that $\mu_{a}^{\mathrm{X}} \nabla_{b} n_{\mathrm{X}}^{a}=\nabla_{b} \Lambda_{\mathrm{X}}$. This is equivalent to the total pressure splitting into partial pressures, and does not hold for all master functions (e.g. in the general entrainment case). We know (see, e.g., [12]) that a general strong form of a system of hyperbolic equations is equivalent, in general, to an infinite number of distinct weak forms, each having different shock propagation speeds. Therefore in the absence of additional physical input (mathematically, the entropy function associated with the system) or an appropriate first principles derivation of a balance law form, there are no uniquely defined discontinuous solutions of (3). The derivation of a suitable entropy satisfying balance law form is required future work before multifluid systems with shocks can be studied.

\section{2. $3+1$ decomposition}

As a test-bed we will consider flat spacetimes in standard plane symmetric Cartesian coordinates. Using $(t, i)$ to represent the time and spatial coordinates, the continuity equation (1) becomes

$$
\partial_{t} n_{\mathrm{X}}^{t}+\partial_{i} n_{\mathrm{X}}^{i}=0
$$

and the Euler equation (3) can be written as

$$
\partial_{t} \mu_{i}^{\mathrm{X}}-\partial_{i} \mu_{t}^{\mathrm{X}}-2 \frac{n_{\mathrm{X}}^{j}}{n_{\mathrm{X}}^{t}} \partial_{[i} \mu_{j]}^{\mathrm{X}}=0 .
$$

\subsection{Numerical implementation}

The nonlinear numerical simulations solve the equations of motion (4-5). Although these describe conservative ideal (multi-species) hydrodynamics, they are not written in balance law form, as discussed in section 2.1. Therefore discontinuous "solutions" will not necessarily be the correct (entropy satisfying) solution, irrespective of the 
numerical techniques employed. For simplicity we will therefore use a basic finite difference discretization of the equations of motion (4-5) and note that the numerical solutions should not be trusted in two regimes: firstly, when the spatial gradients are sufficiently large to appear discontinuous on the grid, and secondly when the solution is dominated by high frequency (relative to the numerical grid) components.

The implementation used here relies on the Method of Lines where the time integration uses either the optimal third order strict stability preserving (SSP) RungeKutta method (see e.g. equation (4.2) of [13]), or the standard fourth order non-SSP Runge-Kutta method (see e.g. equation (12.33) of [14]). The spatial discretization uses central differencing of either second or fourth order. We have tested whether the addition of Kreiss-Oliger dissipation (of either third or fifth order respectively) makes any quantitative difference to either the numerical errors or convergence, or qualitative difference to the onset, growth or saturation of the two-stream instability, and it does not. In all simulations we set the timestep by imposing a CFL constant $\Delta t / \Delta x$ of 0.25 , and have checked that the results are insensitive to the precise value. All models studied here use the shearing box approximation, so periodic boundary conditions are applied to all variables.

The numerical solution of equation (4-5) gives solutions for $n_{\mathrm{X}}^{t}$ and $\mu_{i}^{\mathrm{X}}$. This is sufficient to describe the system completely, but in order to numerically compute the terms for the next update it is necessary to construct the spatial components of the number density flux, $n_{\mathrm{X}}^{i}$. Combining the spatial components (to be computed) with the time components (given from the evolution) we have all components of $n_{\mathrm{X}}^{a}$ from which, given the spacetime metric. we can then compute $n_{\mathrm{X}}^{2}$ and hence, using $(2), \mu_{a}^{\mathrm{X}}$, allowing the evolution to proceed.

To construct the spatial components $n_{\mathrm{X}}^{i}$ given known values for $n_{\mathrm{X}}^{t}$ and $\mu_{i}^{\mathrm{X}}$ two steps are taken. First we note that if the number densities $n_{\mathrm{X}}^{2}$ are assumed known with values $\bar{n}_{\mathrm{X}}^{2}$ then the definition of the conjugate momenta, (2), is a linear system for the spatial components $n_{i}^{\mathrm{X}}$,

$$
\mu_{i}^{\mathrm{X}}=A^{\mathrm{XZ}}\left(\bar{n}_{\mathrm{Y}}^{2}\right) n_{i}^{\mathrm{Z}}
$$

where we have implicitly summed over the species index $\mathrm{Z}$ and the matrix $A$ is given by the derivatives of the master function $\Lambda$ evaluated at the (assumed known) values $\bar{n}_{\mathrm{Y}}^{2}$.

In the second step we take the spatial components constructed by the solution of the linear system in (6) and construct, using the known spacetime metric, $n_{\mathrm{X}}^{a}\left(\bar{n}_{\mathrm{Y}}^{2}\right)$. This gives an independent calculation of the number densities $n_{\mathrm{X}}^{2}$. Obviously the correct value of $n_{\mathrm{X}}^{2}$ is given by the root of the function

$$
f_{\mathrm{X}}\left(\bar{n}_{\mathrm{Y}}^{2}\right)=\bar{n}_{\mathrm{X}}^{2}-n_{\mathrm{X}}^{2}\left(\bar{n}_{\mathrm{Y}}^{2}\right) \text {. }
$$

The existence and uniqueness of such a root places constraints on the master function that we neither attempt to clarify nor check. A standard root-finding procedure, such as a Newton solver, can be employed when a root exists.

The robustness of the root-finding procedure is the main weakness in our numerical implementation. Standard algorithms are sensitive to the initial guess, and for a multi-dimensional root-find as required here there is no general procedure to locate a region in parameter space within which a root must lie. In our implementation the initial guess is given by the value from the previous timestep, with some ad hoc fallback procedures should this fail. This method is successful for moderate fluid velocities (typically up to $|v| \sim 0.8$ ) but fails rapidly as any velocity nears the speed of light. 


\section{Linearized solution}

To study the time domain behaviour of the multifluid system, and as a comparison test for the nonlinear simulations, we construct linearized solutions of two coupled fluids on a $2 L$ periodic domain $[-L, L]$ in one spatial dimension. The restrictions on the number of fluids and spatial dimensions can be dropped in the following analysis at the cost of substantially complicating the explicit calculation of the final solution.

\subsection{Linearized system}

Start by restricting the equations of motion (4-5) to one spatial dimension $x$, which after linearizing gives the system

$$
\begin{aligned}
& \partial_{t} \delta n_{\mathrm{X}}^{t}+\partial_{x} \delta n_{\mathrm{X}}^{x}=0, \\
& \partial_{t} \delta \mu_{x}^{\mathrm{X}}-\partial_{x} \delta \mu_{t}^{\mathrm{X}}=0 .
\end{aligned}
$$

Note that, from the definition of the conjugate momenta given in (2), we have (see [11])

$$
\delta \mu_{a}^{\mathrm{X}}=\mathcal{M}_{a b}^{\mathrm{XZ}} \delta n_{\mathrm{Z}}^{b}
$$

where here, and in the future, $\mathrm{Z}$ is an abstract species index to be summed over, and where the matrices can be given explicitly (e.g. [11, section 11.3]).

We note that the linearized system can be written as

$$
\begin{array}{ll}
\partial_{t} \delta n_{\mathrm{X}}^{t}+\partial_{x} \delta n_{\mathrm{X}}^{x} & =0, \\
\mathcal{M}_{x x}^{\mathrm{XZ}} \partial_{t} \delta n_{\mathrm{Z}}^{x}-\left(\mathcal{M}_{x t}^{\mathrm{XZ}}+\mathcal{M}_{t x}^{\mathrm{XZ}}\right) \partial_{x} \delta n_{\mathrm{Z}}^{x}-\mathcal{M}_{t t}^{\mathrm{XZ}} \partial_{x} \delta n_{\mathrm{Z}}^{t}=0 .
\end{array}
$$

We will not use this form in the linearized solution, but will use it to construct numerical solutions, using the techniques of section 2.3, for direct comparison with the nonlinear results.

\subsection{Transformed system}

We now use a Fourier-Laplace analysis to solve the linearized system. The spatial Fourier transform converts functions of $x$ to functions of frequency $\omega$. Within frequency space the individual modes of the linearized solutions do not couple, simplifying the analysis and highlighting key features of the physics to be discussed later. The Laplace transform in time, mapping $t$ to a transformed variable $s$, is here only used to simplify the resulting coupled system to an algebraic form that is straightforwardly solved.

Firstly taking the discrete Fourier transforms by writing

$$
\delta n_{\mathrm{X}}^{a}=\sum_{k=0}^{k_{\max }} \tilde{n}_{\mathrm{X},[k]}^{a} \exp \left[\mathrm{i} \omega_{k} x\right]
$$

where $\omega_{k}=\pi k / L$. Then the Laplace transform (denoted $\mathcal{L}(f(t, \omega))=F(s, \omega)$ ), for which we use the notation

$$
N_{\mathrm{X},[k]}^{a}=\mathcal{L}\left[\tilde{n}_{\mathrm{X},[k]}^{a}\right],
$$

leads to the fully transformed equations

$$
\begin{array}{ll}
s N_{\mathrm{X},[k]}^{t}+\mathrm{i} \omega_{k} N_{\mathrm{X},[k]}^{x} & =\tilde{n}_{\mathrm{X},[k]}^{t}(t=0), \\
s\left[\mathcal{M}_{a x}^{\mathrm{XZ}} N_{\mathrm{Z},[k]}^{a}\right]-\mathrm{i} \omega_{k}\left[\mathcal{M}_{a t}^{\mathrm{XZ}} N_{\mathrm{Z},[k]}^{a}\right] & =\mathcal{M}_{a x}^{\mathrm{XZ}} \tilde{n}_{\mathrm{Z},[k]}^{a}(t=0) .
\end{array}
$$




\subsection{Solution}

Combining the results from equation $(13 a-13 b)$, the solution for $N_{\mathrm{X},[k]}^{x}$ follows from the linear system

$$
A^{\mathrm{XZ}} N_{\mathrm{Z},[k]}^{x}=\hat{V}^{\mathrm{X}}
$$

where the components of the matrix $A$ is given by

$$
A^{\mathrm{XZ}}=s^{2} \mathcal{M}_{x x}^{\mathrm{XZ}}-s i \omega_{k}\left(\mathcal{M}_{t x}^{\mathrm{XZ}}+\mathcal{M}_{x t}^{\mathrm{XZ}}\right)+\omega_{k}^{2} \mathcal{M}_{t t}^{\mathrm{XZ}}
$$

and the components of the vector $\hat{V}$ by

$$
\hat{V}^{\mathrm{X}}=s \mathcal{M}_{x x}^{\mathrm{XZ}} \tilde{n}_{\mathrm{Z},[k]}^{x}(t=0)+\mathrm{i} \omega_{k} \mathcal{M}_{t t}^{\mathrm{XZ}} \tilde{n}_{\mathrm{Z},[k]}^{x}(t=0) .
$$

The solution for $N_{\mathrm{X},[k]}^{t}$ follows from $(13 a)$. Given these solutions we can invert the Laplace and Fourier transforms to construct $\delta n_{\mathrm{X}}^{a}$ at any future time. By just inverting the Laplace transform we can construct the solution in frequency space at any future time.

\subsection{The instability}

We note that the qualitative change from a stable two-fluid system to an unstable one can easily be seen with this linearized solution. In the formal solution given by (14) the inverse of the matrix with coefficients $A^{\mathrm{XZ}}$ can be written

$$
(A)^{-1}=\frac{\operatorname{adj} A}{\alpha \prod_{i=1}^{4}\left(s-\mathrm{i} \omega_{k} r_{i}\right)}
$$

where the denominator, a quartic polynomial in $s$, is the determinant of $A^{\mathrm{XZ}}$. This form is chosen so that the roots $r_{i}$ are independent of the frequency $\omega_{k}$.

On inverting the Laplace transform to construct the solution for the linearized number currents in either space or frequency, we find that the time dependent behaviour is encoded in exponentials of the form $\exp \left(\mathrm{i} \omega_{k} r_{i} t\right)$. In the uncoupled case these roots are real and are directly related to the sound speed, leading to the standard wave propagation solution. In the coupled case it is possible for the roots to have non-vanishing imaginary part, which (as in this case the imaginary solutions would appear in conjugate pairs) would lead to an unstable, exponentially growing solution. In particular we note that, when unstable, the growth rate is linearly related to the frequency $\omega_{k}$ as expected. This precisely mirrors previous calculations (e.g. [4]) where the stability was usually found more straightforwardly in terms of the dispersion relation.

\section{Results}

In what follows we shall always use an equation of state inspired by [9] and [15] and encompassing the key expected behaviour. First note ([15]) that the Lorentz factor of one fluid in the frame of the other is

$$
W_{12}=-\frac{n_{1}^{a} n_{2}^{b}}{n_{1} n_{2}} g_{a b}=\frac{n_{12}^{2}}{n_{1} n_{2}}=\left(1-\Delta^{2}\right)^{-1 / 2},
$$

implying that $\Delta$, defined by

$$
\Delta^{2}=1-\left(\frac{n_{1} n_{2}}{n_{12}^{2}}\right)^{2}
$$


Table 1. The parameters used in the simulations below, based on the master function given in (20). In each case one parameter, indicated with $p$, controls the coupling between the two species. In the pure entrainment case where $\kappa_{12}=0$ the values of $\sigma_{\mathrm{X}}$ are irrelevant.

\begin{tabular}{lllllllllll}
\hline & $m_{1}$ & $m_{2}$ & $\kappa_{1}$ & $\kappa_{2}$ & $\gamma_{1}$ & $\gamma_{2}$ & $\kappa_{12}$ & $\sigma_{1}$ & $\sigma_{2}$ & $\kappa_{\Delta}$ \\
\hline Entrainment & 1 & 1 & $\frac{1}{2}$ & $\frac{1}{2}$ & $\frac{8}{5}$ & $\frac{9}{5}$ & 0 & N/A & N/A & $p$ \\
Chemical coupling & 0 & 0 & $2^{-1 / 2}$ & 1 & 1 & $\frac{4}{3}$ & $p$ & 1.1 & 1.1 & 0 \\
\hline
\end{tabular}
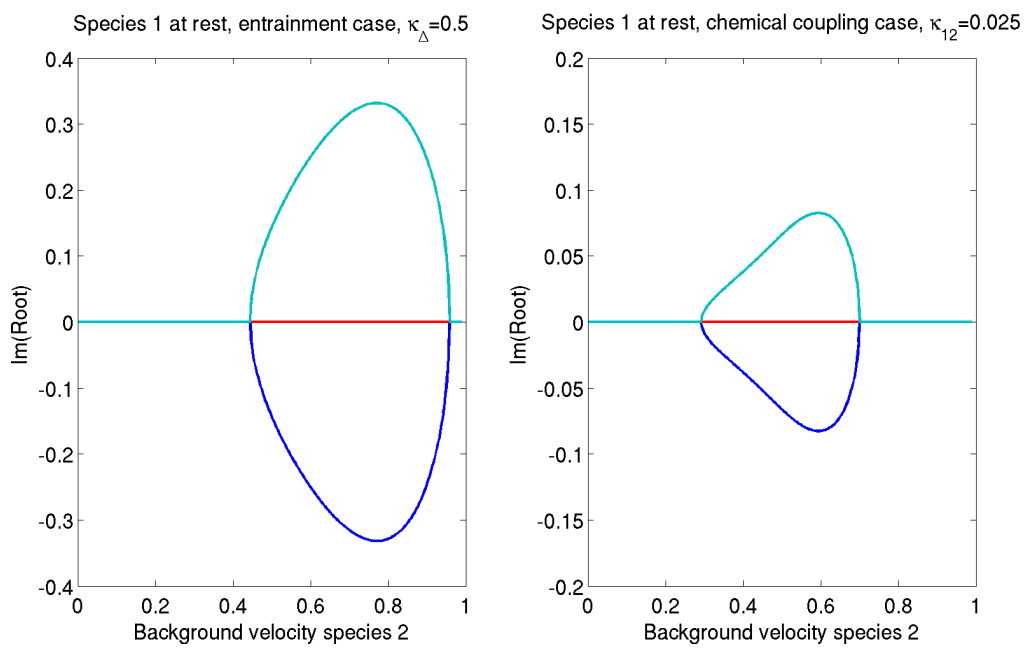

Figure 1. The two-stream instability acts when the roots of the determinant of a particular matrix (see (17) and accompanying text) have non-vanishing imaginary part. The left plot shows the entrainment case, and the right the chemical coupling case - the full parameters are outlined in the text and table 1. For this particular choice there is little qualitative difference in the instability window.

encodes the velocity difference between the two fluids. Then we use a master function with general form

$$
-\Lambda\left(n_{1}^{2}, n_{2}^{2}, n_{12}^{2}\right)=\sum_{\mathrm{X}=1}^{2}\left(m_{\mathrm{X}} n_{\mathrm{X}}+\kappa_{\mathrm{X}} n_{\mathrm{X}}^{\gamma_{\mathrm{X}}}\right)+\kappa_{12} n_{1}^{\sigma_{1}} n_{2}^{\sigma_{2}}+\kappa_{\Delta} \Delta .
$$

We would approximately expect the $\kappa_{\Delta}$ term to encode the entrainment effects, and the $\kappa_{12}$ term to encode the chemical coupling.

We look at the entrainment and chemical coupling cases separately, insofar as this separation makes sense at the nonlinear level. The precise parameters employed are given in table 1 . In the pure entrainment case the strength of the coupling is determined by the parameter $\kappa_{\Delta}$. For the pure chemical coupling case we consider parameters relevant to cosmology (see [9]), where the strength of the coupling is determined by the parameter $\kappa_{12}$. The chemical coupling case also checks that the methods work in the limit $m_{\mathrm{X}} \rightarrow 0$ as expected. Figure 1 shows the instability window for these representative cases where the background is given by the simple choice $n_{\mathrm{X}}^{2}=1$. 

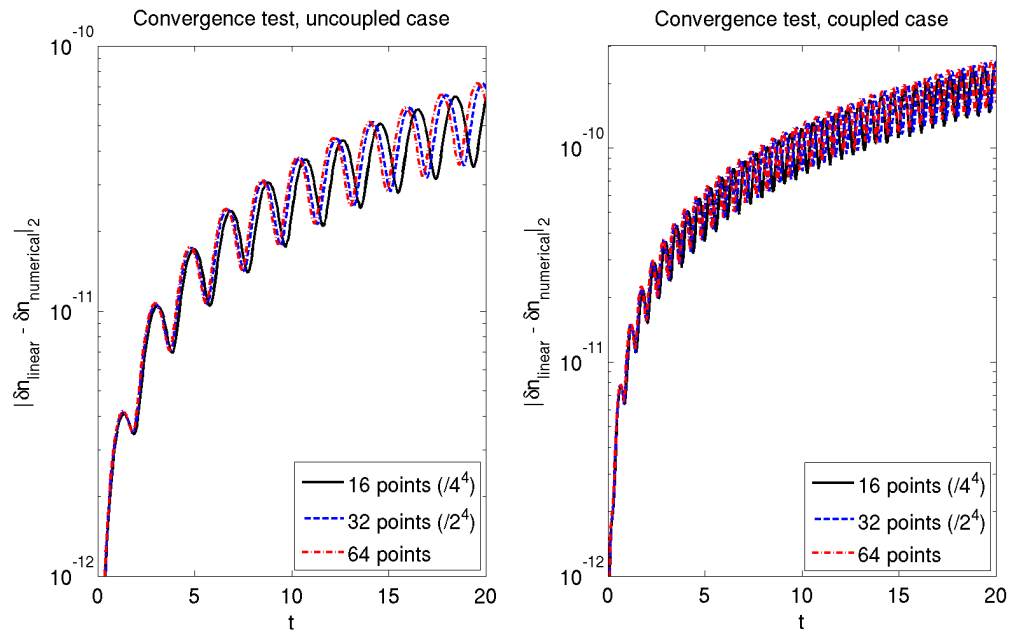

Figure 2. Convergence tests of the nonlinear code against the linearized solution. In both cases the nonlinear code is fully fourth order accurate and the curves are scaled to show the fourth order convergence. The entrainment case is shown, and in both cases the perturbation is a sine wave period $2 \pi$, amplitude $\delta=10^{-6}$, in $n_{1}$. In the left panel the fluids are uncoupled, $\kappa_{\Delta}=0$, and both are at rest in the background. In the right panel the fluids are coupled with $\kappa_{\Delta}=0.5$ and the first fluid also has a non-trivial background velocity of 0.1 whilst the second is at rest.

The initial data chosen here is always written as a perturbation about a background, even in the nonlinear case. We write (in $1+1$ dimensions)

$$
n_{\mathrm{X}}^{a}=n_{\mathrm{X}} W_{\mathrm{X}}\left(-1, v_{\mathrm{X}}, 0,0\right), \quad W_{\mathrm{X}}=\left(1-v_{\mathrm{X}}^{2}\right)^{-1 / 2}
$$

where $W_{\mathrm{X}}$ is the Lorentz factor for the $\mathrm{x}$ species. Perturbations about the background to either $n_{\mathrm{X}}$ or $v_{\mathrm{X}}$ (or both) have been tested. In what follows we shall concentrate on perturbations in $n_{\mathrm{X}}$, as no qualitative difference between the cases has been found. In the majority of the cases we focus on sinusoidal perturbations of amplitude $\delta$, with different frequencies compatible with the size of the domain. These show clean behaviour at very low numerical resolution and are easy to study. Other perturbations (such as the Gaussian shown below) have been tested with no qualitative change in the key features, but may be less flexible (because of the periodic boundaries) and may require higher resolution (due to the formation of steep gradients without forming discontinuities).

\subsection{Convergence}

Firstly we use the linearized solution outlined in section 3 to benchmark the nonlinear code in the stable regime. This is only possible for a certain range of initial perturbation amplitudes, outside of which the convergence of the error is limited by nonlinear effects or floating point precision. In figure 2 we show simple convergence tests for a single mode perturbation in the entrainment case - similar results are seen in the chemical coupling case. The master function parameters are as detailed above. In addition, for the uncoupled case we have $\kappa_{\Delta}=0$ and for the coupled case we choose $\kappa_{\Delta}=1 / 2$ - again, similar results are seen for all values of $\kappa_{\Delta}$ and $\kappa_{12}$ in the 

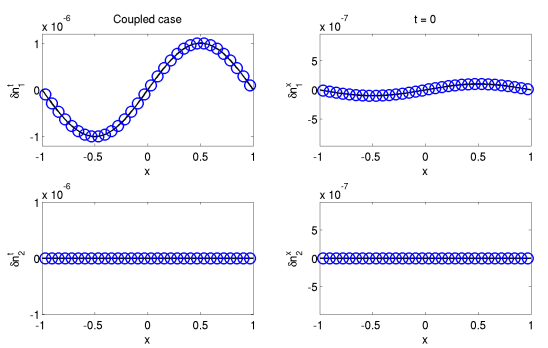

$\times 10^{-7}$

5.
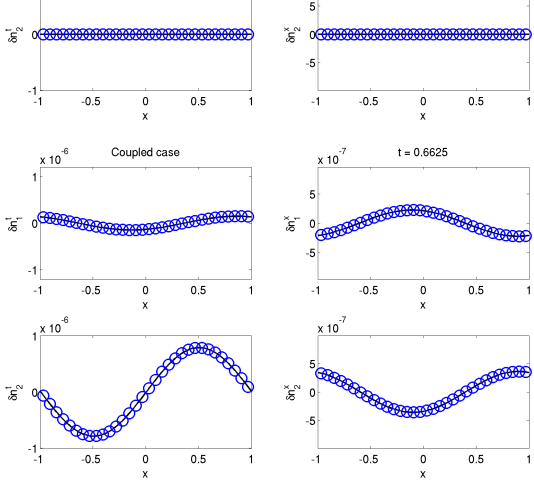
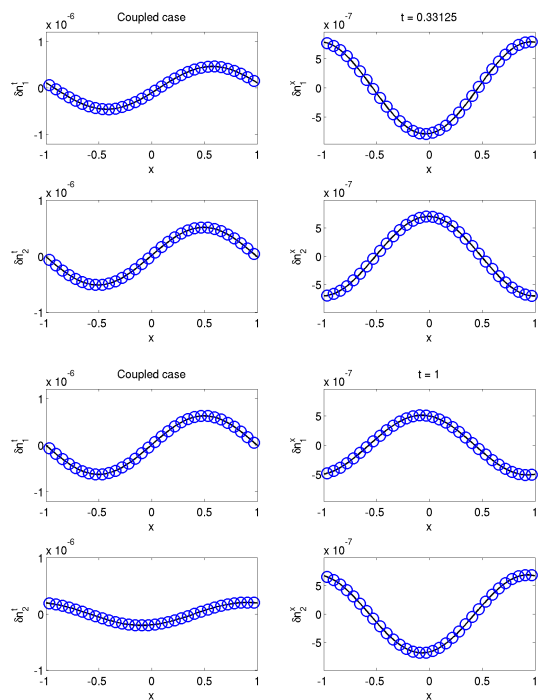

Figure 3. A direct comparison of the time evolution of the number density components in the coupled case used in the convergence plot in figure 2 . The linearized solution is given by the solid black lines, and the nonlinear numerical evolution (using only 32 points) is given by the blue circles. With these parameters we see that the fluids are fully coupled within one crossing time, but that the profiles retain the general shape given by the initial perturbation.

ranges considered here. The uncoupled case is the simplest possible - both fluids are at rest in the background with $n_{\mathrm{X}}=1$. In the coupled case a velocity difference is imposed by giving the first fluid a velocity of 0.1 . The perturbation is imposed only in the first fluid and is a simple sine perturbation, period $2 \pi$, amplitude $\delta=10^{-6}$, in the number density $n_{1}$. Similar results are seen when the initial perturbation is a Gaussian, although considerably higher resolution is required to resolve the spatial gradients.

We note that the results converge as expected for low resolutions. At higher resolution we typically see results that are not perfectly convergent, either due to the tiny nonlinear couplings starting to dominate over the linear effects, or because the numerical error is affected by floating point precision. Where the dominant effect is the nonlinear couplings we still see the expected self-convergence of the nonlinear code.

A direct comparison of the components of the number densities is given in figure 3 . Over the period of approximately one crossing time we see the excellent agreement between the linearized solution and the nonlinear evolution, even at extremely low numerical resolution. The fluids rapidly couple with this choice of entrainment parameter - within this time, which is approximately one crossing time, the amplitude of the second fluid is comparable to that of the first which contained the perturbation. The form of the perturbation is also retained through the evolution. 


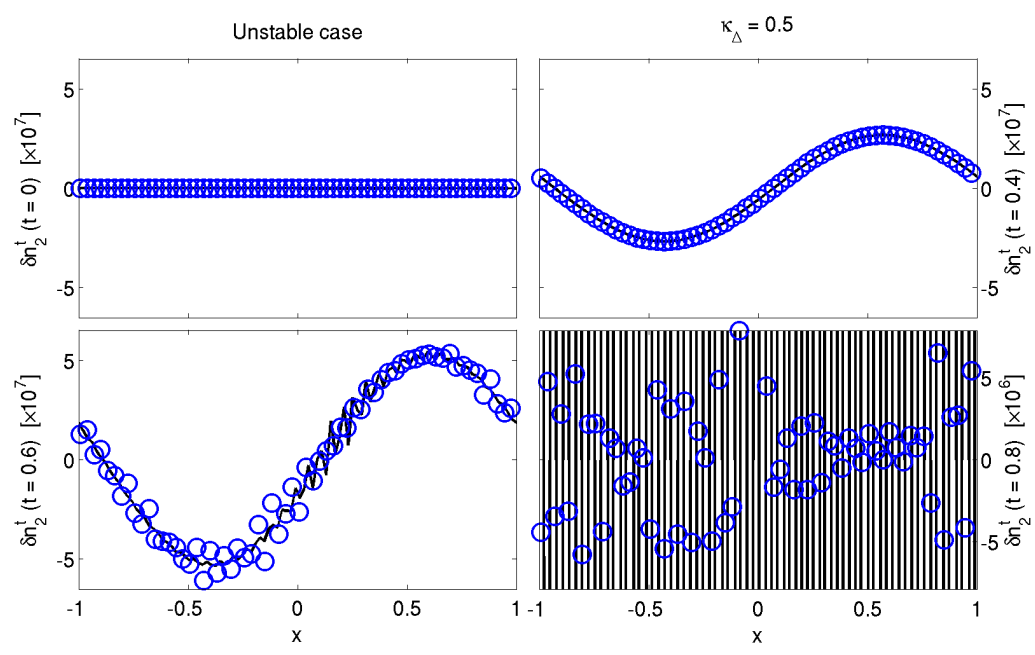

Figure 4. A direct comparison of the time evolution of a number density component in the coupled case within the instability window for the two-stream instability. The linearized solution is given by the solid black line, and the nonlinear numerical evolution (using 128 points - every second shown) is given by the blue circles. This is the entrainment case with $\kappa_{\Delta}=0.5$ and a background velocity difference of 0.6. The instability grows rapidly in both linear and nonlinear case; to compare, the linear solution seeds all modes up to $k=64$ with small random noise. In the early stages the behaviour closely follows the stable case shown in figure 3. By $t=0.6$ (bottom left panel) the instability is visible in the high-frequency oscillations for both the linear solution (solid line) and the nonlinear numerical solution (circles). By $t=0.8$ (bottom right panel) the instability is clearly dominating both the nonlinear numerical solution and the linear solution, and the full extent of the oscillations no longer fit within the scale of the plot. The two-stream instability grows fastest in the highest frequency modes, seeded by hand in the linear case and by numerical error in the nonlinear case. The exponential growth is not spatially localized and behaves in qualitatively the same fashion in all components of all species.

\subsection{Instability growth}

We first consider the entrainment case with $\kappa_{\Delta}=1 / 2$. As an illustration we look at a case where the first fluid is perturbed with a simple sine wave as before, but is at rest, whilst the second fluid is unperturbed but has a velocity of 0.6 in the background so that the two-stream instability acts. Representative results are shown in figure 4 and figure 5 . We note first that the space-time development of the instability appears to be (a) dominated by the high frequency components, (b) not spatially localized to the perturbation (as shown in particular when the initial perturbation is a narrow Gaussian, as in figure 5), and (c) closely following the linearized solution, where we assume that the linearized solution is "seeded" by constant frequency low amplitude perturbations in all modes in addition to the perturbation used in the numerical simulation.

4.2.1. Time-frequency behaviour: linear case More detail can be seen when the solution is studied in time-frequency space. These results, however, mix numerical and nonlinear effects. In order to disentangle these effects as far as possible we first 


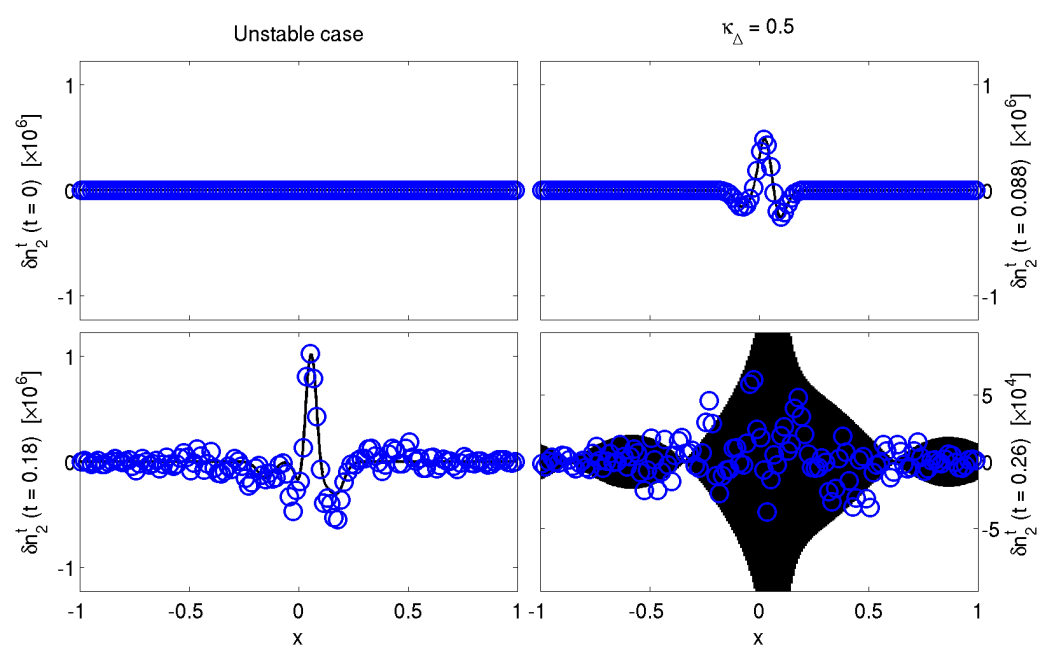

Figure 5. A direct comparison of the time evolution of a number density component in the coupled case within the instability window for the two-stream instability. The linearized solution is given by the black line, and the nonlinear numerical evolution (using 512 points - every fourth shown) is given by the blue circles. This is the entrainment case with $\kappa_{\Delta}=0.5$ and a background velocity difference of 0.6 , as in figure 4, but here the initial perturbation is a Gaussian, hence the higher resolution required. The instability grows rapidly in both linear and nonlinear case; to compare, the linear solution seeds all modes up to $k=256$.

consider solely the linearized case, as shown in figure 6 . In the linear case we expect the different frequency modes to behave independently. The change in power of a given frequency mode with time will therefore illustrate only effects due to the twostream instability where relevant and the numerical method employed. The linearized solution, as constructed in section 3.3, is shown in the left panel and shows simple characteristic behaviour, with power-law behaviour in the instability growth time scale. This behaviour is not replicated in the numerical simulations of the linear system, shown in the right panel. This must be purely due to the numerics used. This numerical effect can be modelled analytically.

Following the work of Lele [16] we note that the effect of the finite difference scheme, when approximating spatial derivatives, is equivalent to replacing the exact Fourier transform relation $\partial_{x} f \rightarrow \mathrm{i} \omega \hat{f}$ with $\partial_{x} f \rightarrow \mathrm{i} \omega^{\prime} \hat{f}$, where $\omega^{\prime}$ encodes how accurately the numerical method captures modes of a given frequency. For the centred fourth order differencing that we focus on here a straightforward calculation (or appropriately applying the general results of [16]) gives

$$
\tilde{\omega}^{\prime}(\tilde{\omega})=\frac{8 \sin (\tilde{\omega})-\sin (2 \tilde{\omega})}{6} .
$$

Here we use $\tilde{\omega}=\pi \omega / k_{\max }$ to scale to Lele's units. Repeating the linear analysis using $\omega^{\prime}$ in place of $\omega$ throughout leads to the results in the central panel of figure 6 . The match between these adjusted "exact" results and the results from numerical simulation in the right panel of figure 6 is extremely good. The remaining minor differences are likely due to the choice of using uniform amplitude "noise" to seed the analytic calculation at higher frequencies, as compared to the numerical simulation where the noise likely appears from floating point round-off error. This assumption 


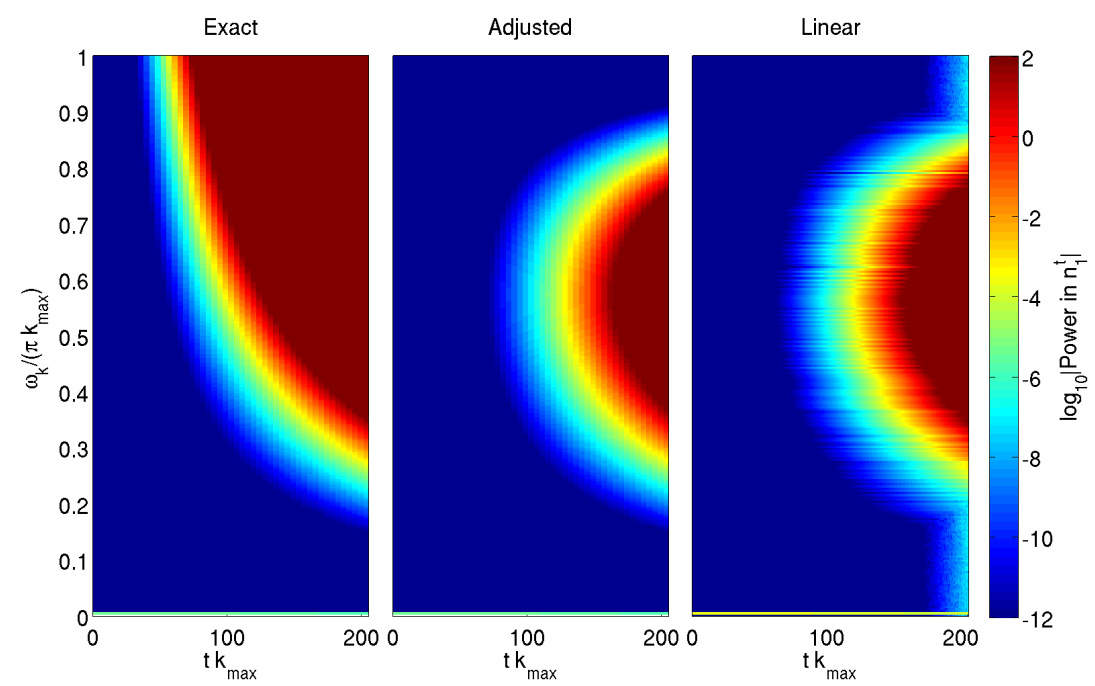

Figure 6. A time-frequency plot of the growth of the instability in the linearized case. Both time and frequency are scaled to be resolution independent - the numerical simulation shown uses 1024 points $\left(k_{\max }=512\right)$. The norm of the complex component of the discrete Fourier Transform of $\delta n_{1}^{t}$ is shown - the result for other components and quantities is qualitatively the same. The left plot shows the behaviour for the linearized solution. As expected the highest frequencies blow up the fastest. The central plot shows the behaviour for the linearized solution, adjusted as in (22) to take into account the behaviour of the spatial differencing scheme. This would be the expected behaviour from a numerical evolution of the linearized equations. The right plot shows the results from the numerical linear evolution. We see that the adjustment for the spatial differencing scheme completely explains the numerical behaviour, suppressing the growth of the instability at high frequencies.

is supported by the amplitude of the seed noise used in this comparison, which is $\approx 10^{-16} \times \delta$ : the relative floating point accuracy of the linearized perturbation.

4.2.2. Time-frequency behaviour: nonlinear case We next sketch our expectations of how the nonlinear effects would modify the behaviour. Considering a single fluid and taking solely the discrete Fourier Transform of the nonlinear system of equation (4-5), we would expect the nonlinear modes to satisfy coupled equations of the qualitative form

$$
\partial_{t} \tilde{n}_{[k]}^{a}+A_{[k]} \circledast \tilde{n}_{[k]}^{a}=0,
$$

where $\circledast$ is the (cyclic) convolution product. In the linear case the matrix $A_{[k]}$ would only contain the constant (zero frequency, $k=0$ ) term. In the nonlinear case the terms depend on the master function and the data - in other words, on the non-trivial $\tilde{n}_{[k]}^{a}$.

With initial data dominated by say two frequencies $k_{1}$ and $k_{2}$ the convolution couples modes at the harmonics with frequencies $a k_{1}+b k_{2}$ with $a, b$ integers. For the typical initial data used here the dominant frequencies will be from the background $(k=0)$ and the initial perturbation with frequency $k_{0}$, leading to harmonics at integer multiples of $k_{0}$. This effect can be clearly seen in numerical nonlinear simulations as shown in figure 7 . In this case the background is chosen so that the velocity difference 

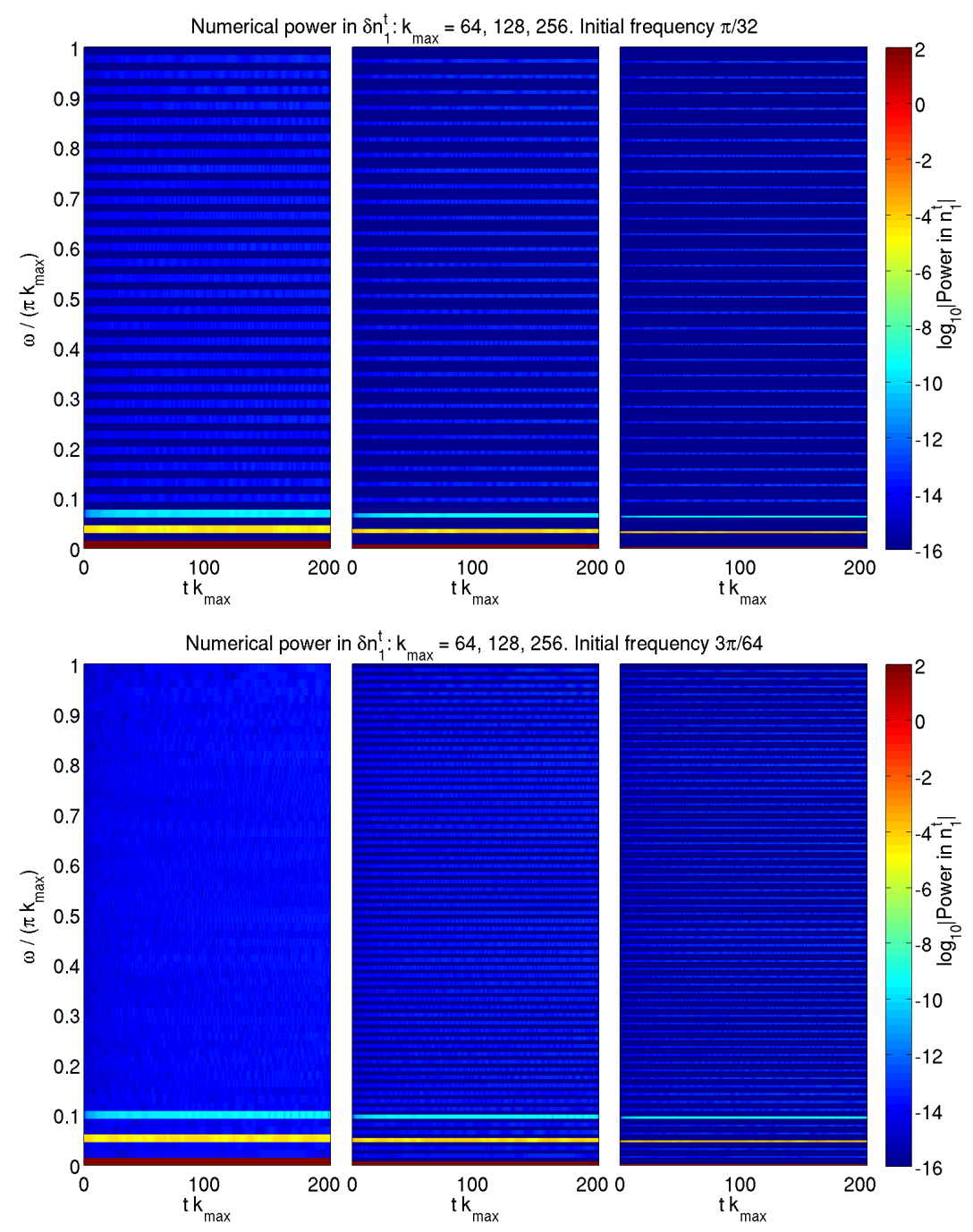

Figure 7. A time-frequency plot showing the harmonic coupling expected in the nonlinear case. As the grid resolution (and hence frequency maximum $k_{\max }$ ) is varied the initial perturbation is modified to give a constant frequency with respect to the grid. In all cases the perturbation has a single frequency. The nonlinear coupling immediately excites all the higher harmonics, although only the lowest harmonic (at twice the frequency of the initial perturbation) is excited at a level that would be visible. The initial parameters are chosen such that the fluids couple but the two-stream instability is not acting.

is 0.1 so the two-stream instability does not act. The fluids couple and the higher harmonics are excited, but only the frequencies associated with the background, the initial perturbation and the first harmonic would be noticeable in the spatial snapshots.

Once the background is modified to allow the two-stream instability to act we can see both the numerical suppression of the high frequency modes (as shown in the linear case in figure 6) and the nonlinear coupling of the higher harmonics (as shown in the stable case in figure 7). Figure 8 repeats the calculation exciting higher 
harmonics as in figure 6 , but the background is modified so that the velocity difference is 0.6 and the two-stream instability acts. Whilst all modes should be excited to some degree by numerical error and nonlinear couplings, only those that are coupled via harmonic overtones are excited sufficiently to survive numerical truncation effects and hence grow exponentially - only by increasing the amplitude to the point where the subleading order couplings are significant (which here happens when $\delta \sim 10^{-3}$ ) will these modes show exponential growth. The figure then shows precisely these excited modes growing exponentially as expected, initially independently of the other modes, with the suppression at higher frequency qualitatively matching the behaviour seen in the linear case.

We can now look at the original case, studied for the linear problem in figure 6 . Here the only mode initially excited is the lowest mode on the grid. As seen in figure 9, there is still a qualitative match between the adjusted linear solution and the numerical evolution, even in the nonlinear case. The "bulk" features, including the approximate growth rates and the suppression of the growth at high frequencies, remain the same. Whilst there are also noticeable differences at late times, there is no clear pattern in either the space-time development or the time-frequency growth, except for the increased coupling between the different frequencies at late time.

\subsection{The instability onset}

The parameters chosen for both the entrainment and chemical coupling case have a "window" where the two-stream instability acts, as shown by figure 1. By modifying the relative velocity of the background in the initial data, we can check that the onset of the instability occurs at the same point with the nonlinear code.

In both the entrainment and chemical coupling case we find that, when using small perturbations of amplitude $\delta=10^{-6}$ as above, the onset of the instability (with increasing relative velocity) is, to numerical precision, identical to that predicted from the linear analysis. In the chemical coupling case we can also check the upper edge of the window; that is, the nonlinear results show the instability for $0.29185 \lesssim \Delta v \lesssim 0.69985$, and are stable otherwise. In the entrainment case we are unable to check the upper edge of the window (which is at $\Delta v \approx 0.958$ ) as the velocities required are too large for the nonlinear code to successfully evolve, as the root-finding procedure fails as detailed in section 2.3.

Finally we attempted to produce initial data that would generate shocks before the two-stream instability becomes important, to see whether the nonlinear couplings could ever be expected to be more important than the instability growth. In our experiments, only extremely large initial perturbations $\left(\delta \sim 10^{-1}\right)$, combined with a choice of master function parameters such that the two-stream instability growth is as slow as possible, show signs of characteristic breaking before the two-stream instability sets in. Even in these cases it is possible for the two-stream instability to dominate simply by increasing the grid size, and so admitting grid frequencies that grow sufficiently rapidly. Within this purely ideal hydrodynamics case using the shearing box approximation it does not seem possible to suppress the instability.

\subsection{Higher dimensions}

All the results so far have been restricted to $1+1$ dimensions in the shearing box (periodic boundaries) approximation. We have performed numerical simulations that 

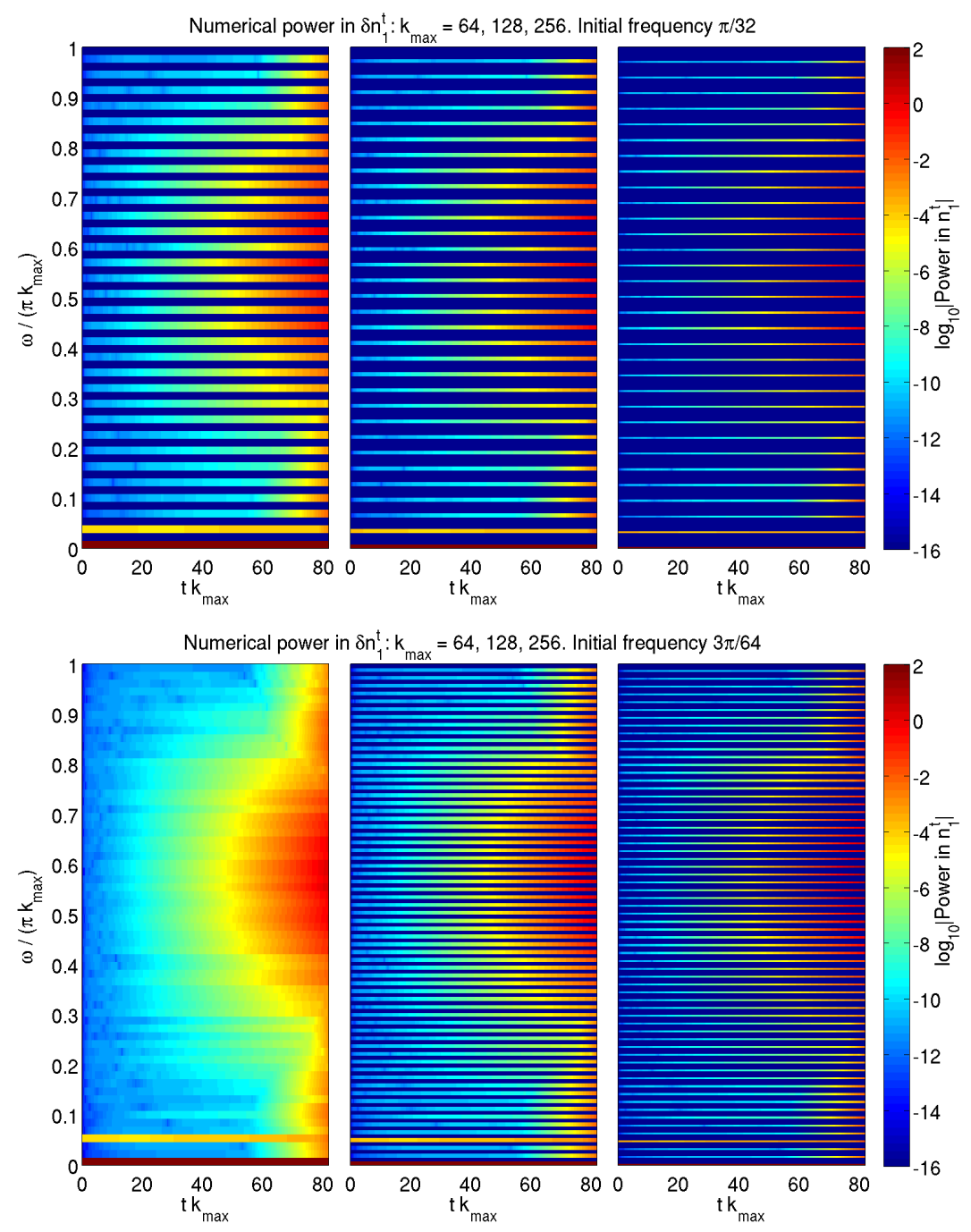

Figure 8. A time-frequency plot of the growth of the instability where the initial frequency (relative to the grid) of the perturbation is held fixed. As the grid size is increased the same number of harmonics are excited by the nonlinear coupling, as in the stable case shown in figure 7. Each excited harmonic then grows exponentially due to the two-stream instability, with the pattern resembling (at early times) the linearized case shown in figure 6). As in the stable nonlinear case in figure 7 there is no visible nonlinear coupling except to the immediate harmonics. In the top row the overtones are always at higher frequencies. The choice of period in the bottom row means that some overtones at lower frequencies are excited, meaning that in the bottom left plot all frequencies on the grid are excited by nonlinear couplings. 


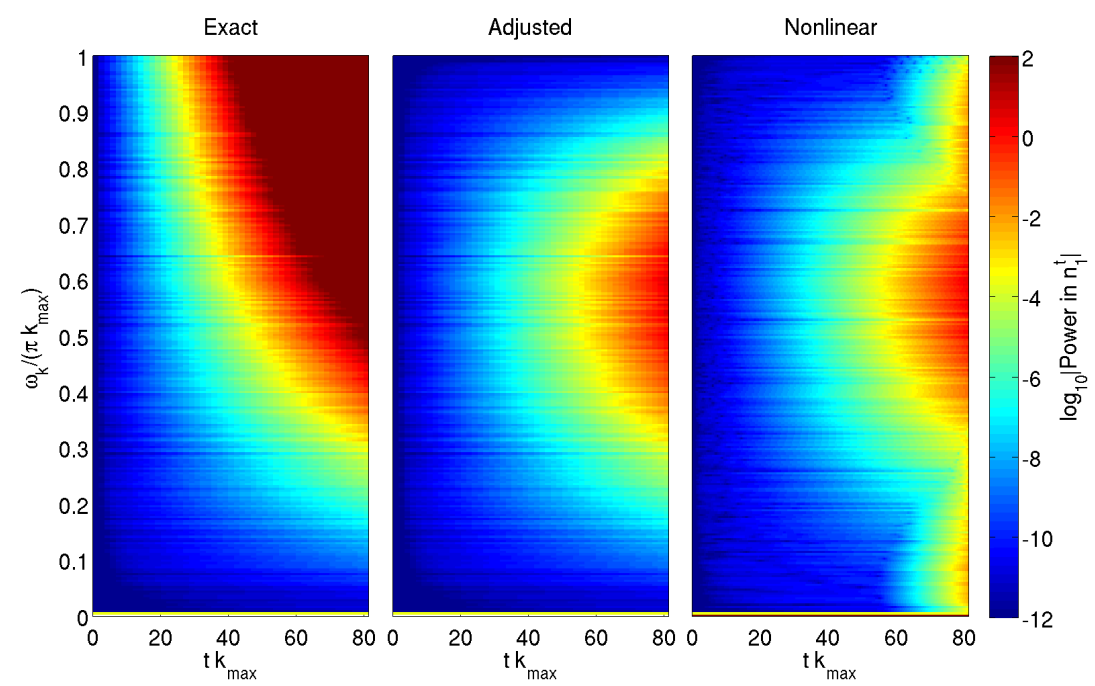

Figure 9. A time-frequency plot of the growth of the instability in the nonlinear case. This figure is the nonlinear equivalent of figure 6 . The linearized and adjusted solutions are seeded with noise from the first timestep of the numerical solution; this is noticeably larger than in the linear case. The qualitative comparison between the adjusted and numerical solution is still reasonable, explaining the suppression of the growth at high frequencies, but the nonlinear coupling does modify the solution significantly.

retain the shearing box approximation in higher dimensions - in particular, detailed comparisons in 2+1 dimensions were calculated. No qualitative differences were found. This can be understood by transforming to the frame of the background flow for one species. In this frame, there are only two possible effects from the additional spatial dimension.

First, the angle between the perturbed flow and the background velocity difference will change the coupling. This is indeed the case: our simulations confirm that the projection of the perturbation onto the velocity difference must be non-zero for the instability to act. However, in this (generic) case, the behaviour of the instability growth is qualitatively identical to the $1+1$ dimensional case.

Second, the coupling between the species should potentially lead to a change in the alignment between the species, modifying the relative velocity between them. This could potentially change the growth of the instability. However, explicit simulations performed by modifying the angle of the perturbation with respect to the relative flow showed no visible difference in, for example, the growth of $\Delta^{2}$. Thus it does not seem possible for purely local hydrodynamic effects, even at the nonlinear level, to modify the behaviour of the instability.

\section{Discussion}

The two-stream instability has been mooted as an explanation for a range of astrophysical applications from GRBs and pulsar glitches to cosmology. We have used numerical simulations to study the nonlinear development of this instability when the species are modelled using coupled relativistic hydrodynamics. 
Our simulations show that the onset of the instability when only the local, purely hydrodynamic behaviour is considered, perfectly matches the predictions of linear theory. The restricted analysis in $1+1$ dimensions is sufficient, provided the initial data is appropriately interpreted, when there is no change in either the spacetime, the external forces, or the coupling parameters. When these restrictions are relaxed, for example in the cosmological case considered by [9] where the expansion of the universe is modelled by varying the coupling parameters, then the instability growth can be curtailed.

The initial growth of the instability is also well described by linear theory, after adjustment to account for the known properties of the numerical simulations. At the very late stages where the instability dominates is there a noticeable difference between the linear and nonlinear behaviour, which has no obvious pattern. The adjustment required to explain the numerical effects illustrates the problems that finite differencing schemes have resolving high grid frequency modes which, as shown by [16], can be reduced without being eliminated by increasing the order of accuracy of the method - even a tenth order method would still not resolve the highest $10 \%$ of the modes. In general the impact of these effects is important when the highest frequency that can be numerically resolved on the grid is physically important. Physically we would expect boundary conditions or non-ideal effects to impose a high frequency cutoff, which then gives the numerical resolution necessary. In the case studied here, the combination of purely conservative ideal multifluids and periodic boundaries means that no such frequency scale exists, and so there will always be effects that cannot be resolved on a finite numerical grid. Spectral type methods could be used to sidestep these problems (as they perfectly capture all frequencies), but have their own well-known problems with the steep gradients expected to form in nonlinear hydrodynamics problems. In the more physically complex cases where a high frequency cutoff should exist it is likely that finite difference methods as used here will still be more straightforward to use apply.

This work is a necessary first step towards generic nonlinear simulations of relativistic multifluids. As multifluid effects such as entrainment are expected to be important in, for example, heat conduction [17], charge conduction and resistivity [18], and neutron superfluids [19], high accuracy nonlinear simulations incorporating detailed microphysics must include multifluids. The main technical barrier to incorporating these effects in current simulations is the lack of either a balance law form or a non-conservative form that explicitly controls entropy at discontinuities. Our future work is aimed at overcoming this technical hurdle before combining the multifluid effects with nonlinear relativistic elasticity [20] and appropriate numerical techniques for interfaces [21] to produce such simulations.

\section{Acknowledgments}

IH and NA acknowledge support from STFC through grant number ST/J00135X/1. GLC acknowledges partial support from NSF through grant number PHYS-0855558.

\section{References}

[1] D. Farley. Two-Stream Plasma Instability as a Source of Irregularities in the Ionosphere. Physical Review Letters, 10(7):279-282, April 1963. 
[2] O. Buneman. Excitation of Field Aligned Sound Waves by Electron Streams. Physical Review Letters, 10(7):285-287, April 1963.

[3] A. Bret, L. Gremillet, and M. E. Dieckmann. Multidimensional electron beam-plasma instabilities in the relativistic regime. Physics of Plasmas, 17(12):120501, 2010.

[4] Lars Samuelsson, Cesar S Lopez-Monsalvo, Nils Andersson, and Gregory L Comer. Relativistic two-stream instability. General Relativity and Gravitation, 42(2):413-433, July 2009.

[5] Irakli S. Nanobashvili. Two-Stream Instability as a Mechanism for Toroidal Magnetic Field Generation in the Magnetosphere of Crab Pulsar. page 7, October 2011.

[6] Peter Sturrock and Markus J. Aschwanden. Flares in the Crab Nebula Driven By Untwisting Magnetic Fields. The Astrophysical Journal, 751(2):L32, June 2012.

[7] Nils Andersson, Gregory L Comer, and Reinhard Prix. Are Pulsar Glitches Triggered by a Superfluid Two-Stream Instability? Physical Review Letters, 90(9):091101, March 2003.

[8] Nils Andersson, Gregory L Comer, and Reinhard Prix. The superfluid two-stream instability. Monthly Notices of the Royal Astronomical Society, 354(1):101-110, October 2004.

[9] Gregory L Comer, Patrick Peter, and Nils Andersson. Cosmological two-stream instability. Physics Letters B, 715(4-5):289-292, September 2012.

[10] Brandon Carter. Relativistic Fluid Dynamics. In Angelo M. Anile and Yvonne Choquet-Bruhat, editors, Relativistic Fluid Dynamics, volume 1385 of Lecture Notes in Mathematics, pages 1-64. Springer Berlin Heidelberg, Berlin Heidelberg, 1989.

[11] Nils Andersson and Gregory L Comer. Relativistic Fluid Dynamics: Physics for Many Different Scales. Living Reviews in Relativity, 10:1-83, 2007.

[12] Randall J LeVeque. Finite Volume Methods for Hyperbolic Problems. Cambridge University Press, Cambridge, 2002.

[13] Sigal Gottlieb, Chi-Wang Shu, and Eitan Tadmor. Strong Stability-Preserving High-Order Time Discretization Methods. SIAM Review, 43(1):89-112, January 2001.

[14] Ender Süli and David F. Mayers. An introduction to numerical analysis. Cambridge University Press, 2003.

[15] Reinhard Prix, Jérôme Novak, and Gregory L Comer. Relativistic numerical models for stationary superfluid neutron stars. Physical Review D, 71(4):1-18, February 2005.

[16] Sanjiva K. Lele. Compact finite difference schemes with spectral-like resolution. Journal of Computational Physics, 103(1):16-42, November 1992.

[17] Nils Andersson and Cesar S Lopez-Monsalvo. A consistent first-order model for relativistic heat flow. Classical and Quantum Gravity, 28(19):195023, October 2011.

[18] Nils Andersson. Resistive relativistic magnetohydrodynamics from a charged multifluids perspective. Physical Review D, 86(4):043002, August 2012.

[19] Nils Andersson, Christian Krueger, Gregory L Comer, and Lars Samuelsson. A minimal model for finite temperature superfluid dynamics. December 2012.

[20] Carsten Gundlach, Ian Hawke, and Stephanie J. Erickson. A conservation law formulation of nonlinear elasticity in general relativity. Classical and Quantum Gravity, 29(1):015005, January 2012.

[21] Stephen T. Millmore and Ian Hawke. Numerical simulations of interfaces in relativistic hydrodynamics. Classical and Quantum Gravity, 27(1):015007, January 2010. 